\title{
Withdrawal-Emergent Dyskinesias following Varenicline Therapy
}

\author{
Brittany A. Toffey, Marcie Rabin and Roger Kurlan*
}

Atlantic Neuroscience Institute, Overlook Medical Center, 99 Beauvoir Avenue, Summit, NJ 07901, USA

\begin{abstract}
Varenicline (Chantix[R]) is a nicotinic acetylcholine receptor partial agonist used to aid smoking cessation. Adverse psychiatric and behavioral effects of the drug are recognized and national drug monitoring has included reports of tardive dyskinesia, but no cases have been described in the literature. We now report the first two cases of vareniclinerelated withdrawal emergent dyskinesias.
\end{abstract}

Keywords: Dyskinesias, verenicline, withdrawal emergent.

\section{INTRODUCTION}

Varenicline (Chantix[R]) is a nicotinic acetylcholine receptor partial agonist used to aid smoking cessation and nicotine addiction. The most common side effects of the drug are nausea, insomnia, and headaches. Adverse psychiatric and behavioral effects of varenicline, such as depression, mania, psychosis and aggression, have also been recognized [1] We now provide the first case reports of involuntary movements occurring upon the withdrawal of varenicline therapy.

\section{REPORT OF CASES}

\section{Case 1}

In her late 40's, this woman was diagnosed as having a mood disorder and anxiety and was placed on lamotrigine, fluoxetine (later replaced with duloxetine), and alprazolam. At age 51 she started varenicline $1 \mathrm{mg} /$ day to help her stop smoking. On varenicline she developed mild action tremor of her hands. Aripiprazole 10mg/day was added for mood stabilization. After approximately 1 year on varenicline, she required a psychiatric hospitalization due to suicidality, depression and the acute onset of hypomanic behavior. During the hospitalization, lithium and buproprion were added, aripiprazole was continued and varenicline was discontinued. Soon after discharge from the hospital, she had the onset of involuntary choreoathetotic movements of her hands, feet, neck, trunk, face, eyelids, mouth, and jaw. Aripiprazole, buproprion, and lithium were stopped. All movements persisted over the next 5 years, but she has achieved symptom control with botulinum toxin injections into the involved cranial muscles. She is taking legal action against the manufacturer of varenicline because of these problems.

*Address correspondence to this author at the Atlantic Neuroscience Institute, Overlook Medical Center, 99 Beauvoir Avenue, Summit, NJ 07901, USA; Tel: 908-522-2089; Fax: 908-522-6147;

E-mail: roger.kurlan@atlantichealth.org

\section{Case 2}

This 65-year-old woman took varenicline $1 \mathrm{mg} /$ day intermittently over the previous 3 years in order to stop smoking. She stopped the drug due to the onset of dizziness, diaphoresis, and 3 weeks later she had the onset of involuntary movements of the jaw and head/neck that appeared to be dystonic tics. These movements have improved with the use of guanfacine $2 \mathrm{mg}$ /day but have persisted for more than 1 year.

\section{DISCUSSION}

Chronic dopamine receptor antagonist antipsychotic drug therapy is known to commonly induce chronic involuntary movements that can persist after stopping the drug, a phenomenon known as tardive dyskinesia. The dyskinesias can be heterogeneous in semiology, including choreoathetosis, dystonia, tics, and other abnormal movements. While the neurobiologic mechanisms of tardive dyskinesia remain unclear, it is thought that chronic dopamine receptor blockade may lead to a compensatory increase in receptor number or sensitivity, resulting in excessive dopaminergic neurotransmission and involuntary movements. Sometimes the movements of tardive dyskinesia first appear upon discontinuation of the drug (withdrawal-emergent dyskinesias), possibly due to the unblocking of upregulated receptors. Our first patient received the antipsychotic drug aripiprazole for less than 1 year prior to the onset of dyskinesias and, although we cannot exclude its etiological role, the short treatment period makes this less likely to be the primary causative agent. The time of onset of her dyskinesias seems to parallel better the time of withdrawal from varenicline. It is also possible that the use and subsequent withdrawal of varenicline may have predisposed the patient to the development of dyskinesias after a fairly brief period of antipsychotic therapy. A period of combined use of an antipsychotic and verenicline may also have predisposed the patient to the occurrence of dyskinesias upon withdrawal of the latter drug. Our second case experienced varenicline-related withdrawal emergent dyskinesias in the absence of any antipsychotic drug treatment. 
Varenicline has been shown to partially stimulate the release of dopamine from its actions at nicotinic acetylcholine receptors [2]. In animals, nicotine increases the production and release of dopamine in the nigrostriatal pathway [3]. Neurochemical models reveal that varenicline is $40 \%-60 \%$ less efficient at stimulating the release of dopamine than is nicotine [2].

Studies have suggested that smokers are more likely to experience tardive dyskinesia than are nonsmokers, although it is known that patients with certain psychiatric conditions, like schizophrenia, that are treated with antipsychotic medications tend to smoke [3]. Also, increased smoking has been correlated with more severe tardive dyskinesia [4]. Since varenicline is used by smokers in trying to stop this habit, this population of patients may be at increased risk of developing drug-induced dyskinesias and perhaps more severe dyskinesias.

According to the eHealthMe database, which compiles information from the Federal Drug Administration about adverse drug effects reported by clinicians, there are reports of tardive dyskinesia in patients who took varenicline [5]. Of the 60,675 patients with a variety of reported side effects in 2013, 25 had tardive dyskinesia [5]. All of the patients with tardive dyskinesia were older than age of 40 [5]. Ours are the first cases of varenicline-related dyskinesias formally described in the literature.

Dopamine receptor antagonists besides antipsychotics, such as metoclopramide and prochlorperazine, are known to be able to cause tardive dyskinesia. While there are reported cases with other drug classes, including antidepressants, anticholinergics, antihistamines and calcium channel blockers, the evidence for direct causation is less clear than that for dopamine antagonists [6]. It appears that the nicotinic partial agonist varenicline is a newly recognized cause of tardive and withdrawal-emergent dyskinesias.

\section{CONFLICT OF INTEREST}

The authors confirm that this article content has no conflicts of interest.

\section{ACKNOWLEDGEMENT}

Toffey-None, Rabin-Speakers bureau for Teva, research support from Ipsen, Kurlan- Speakers bureau for Teva, research support from NIH, Astra-Zeneca, Otsuka, Phytopharm, Psyadon.

\section{AUTHOR ROLES}

Toffey- research conception writing first draft of manuscript, Rabin- research conception, review and critique of manuscript, Kurlan-research conception, review and critique of manuscript.

\section{REFERENCES}

[1] O'Malley P. Quitting smoking without a psychotic break: the potential link between nicotinic acetylcholine receptor partial agonists and neuropsychiatric adverse events. Clin Nurs Spec 2009; 23(1): 13-5.

[2] Rollema H, Chambers LK, Coe JW, et al. Pharmacological profile of the alpha4beta2 nicotinic acetylcholine receptor partial agonist varenicline, an effective smoking cessation aid. Neuropharmacology 2007; 52(3): 985-94.

[3] Yassa R, Lal S, Korpassy A, Ally J. Nicotine exposure and tardive dyskinesia. Biol Psychiatry 1987; 22(1): 67-72.

[4] Diehl A, Reinhard I, Schmitt A, Mann K, Gattaz WF. Does the degree of smoking effect the severity of tardive dyskinesia? A longitudinal clinical trial. Eur Psychiatry 2009; 24(1): 33-40.

[5] Available at: http://www.ehealthme.com/ds/chantix/tardive dyskinesia

[6] Tardive Dyskinesia: summary of a Task Force Report of the American Psychiatric Association. By the Task Force on Late Neurological Effects of Antipsychotic Drugs. Am J Psychiatry 1980; 137(10): 1163-72.

\footnotetext{
Received: January 10, 2014

Revised: March 24, 2014

Accepted: March 25, 2014

(C) Toffey et al.; Licensee Bentham Open.
}

This is an open access article licensed under the terms of the Creative Commons Attribution Non-Commercial License (http://creativecommons.org/licenses/ by-nc/3.0/) 